\title{
Different Cytokine and Chemokine Expression Patterns in Malignant Compared to Those in Nonmalignant Renal Cells
}

\author{
Nadine Gelbrich,, ${ }^{1,2}$ Hannes Ahrend, ${ }^{1}$ Anne Kaul, ${ }^{3}$ Lars-Ove Brandenburg, \\ Uwe Zimmermann, ${ }^{1}$ Alexander Mustea, ${ }^{3}$ Martin Burchardt, ${ }^{1}$ Denis Gümbel, ${ }^{2,5}$ and \\ Matthias B. Stope ${ }^{1}$ \\ ${ }^{1}$ Department of Urology, University Medicine Greifswald, Greifswald, Germany \\ ${ }^{2}$ Department of Trauma, Reconstructive Surgery and Rehabilitation Medicine, University Medicine Greifswald, Greifswald, Germany \\ ${ }^{3}$ Department of Gynaecology and Obstetrics, University Medicine Greifswald, Greifswald, Germany \\ ${ }^{4}$ Department of Anatomy and Cell Biology, RWTH Aachen University, Aachen, Germany \\ ${ }^{5}$ Department of Trauma and Orthopaedic Surgery, BG Klinikum Unfallkrankenhaus Berlin gGmbH, Berlin, Germany
}

Correspondence should be addressed to Matthias B. Stope; matthias.stope@uni-greifswald.de

Received 12 April 2017; Revised 6 June 2017; Accepted 18 June 2017; Published 9 July 2017

Academic Editor: Monica Cantile

Copyright $\odot 2017$ Nadine Gelbrich et al. This is an open access article distributed under the Creative Commons Attribution License, which permits unrestricted use, distribution, and reproduction in any medium, provided the original work is properly cited.

Objective. Cytokines and chemokines are widely involved in cancer cell progression and thus represent promising candidate factors for new biomarkers. Methods. Four renal cell cancer (RCC) cell lines (Caki-1, 786-O, RCC4, and A498) and a nonmalignant renal cell line (RC-124) were examined with respect to their proliferation. The cytokine and chemokine expression pattern was examined by a DNA array (Human Cytokines \& Chemokines RT ${ }^{2}$ Profiler PCR Array; Qiagen, Hilden, Germany), and expression profiles were compared. Results. Caki-1 and 786-O cells exhibited significantly increased proliferation rates, whereas RCC4 and A498 cells demonstrated attenuated proliferation, compared to nonmalignant RC-124 cells. Expression analysis revealed 52 cytokines and chemokines primarily involved in proliferation and inflammation and differentially expressed not only in malignant and nonmalignant renal cells but also in the four RCC cell lines. Conclusion. This is the first study examining the expression of 84 cytokines and chemokines in four RCC cell lines compared to that in a nonmalignant renal cell line. VEGFA, NODAL, and BMP6 correlated with RCC cell line proliferation and, thus, may represent putative clinical biomarkers for RCC progression as well as for RCC diagnosis and prognosis.

\section{Introduction}

Renal cell cancer (RCC) represents the deadliest neoplasm of the urinary tract, due to the fact that a majority of patients are diagnosed at a very advanced stage $[1,2]$. Tumor progression is based on various molecular mechanisms, and thus, further examinations of RCC tumor biology are necessary in order to understand the progression and, furthermore, to identify novel biomarkers for diagnosis and prognosis of RCC $[1,3-5]$.

Among other mechanisms, cytokines and chemokines are suspected to play a crucial role in proliferation and progression of various malignancies. For instance, CCL11 controls cancer cell growth and invasion in ovarian cancer and CCL21 as well as CCR7 represent pivotal regulators of bladder cancer progression [6, 7]. Moreover, IL17, as a representative of interleukins, is associated with lung and colorectal cancer progression and predicts poor prognosis in breast cancer [8-10]. Even though some studies suggested an impact in RCC, the role of cytokines and chemokines in RCC progression is poorly understood $[1,11,12]$. From a clinical point of view, cytokine and chemokine secretion into the blood stream makes them very suitable as noninvasive clinical markers.

In the study presented here, we used an RCC model system consisting of four malignant RCC cell lines compared to a nonmalignant renal cell line. After classification of the RCC cells in cell lines of high and low cell growth rates, a transcriptional profiling specific for 84 cytokines and chemokines (Table 1) was carried out and compared to the 
TABLe 1: Human Cytokines \& Chemokines RT ${ }^{2}$ Profiler PCR Array (Qiagen). Abbreviation (symbol) and full name/function (description) of the 84 cytokines and chemokines analyzed by the PCR array. The column "detectable" indicates whether the factor was detected (+) or not detected (-) within this study.

\begin{tabular}{|c|c|c|c|}
\hline Number & Symbol & Description & Detectable \\
\hline \multicolumn{4}{|c|}{ Chemokines } \\
\hline 1 & C5 & Complement component 5 & + \\
\hline 2 & CCL1 & Chemokine (C-C motif) ligand 1 & - \\
\hline 3 & CCL2 & Chemokine (C-C motif) ligand 2 & + \\
\hline 4 & CCL3 & Chemokine (C-C motif) ligand 3 & - \\
\hline 5 & CCL5 & Chemokine (C-C motif) ligand 5 & + \\
\hline 6 & CCL7 & Chemokine (C-C motif) ligand 7 & + \\
\hline 7 & CCL8 & Chemokine (C-C motif) ligand 8 & - \\
\hline 8 & CCL11 & Chemokine (C-C motif) ligand 11 & + \\
\hline 9 & CCL13 & Chemokine (C-C motif) ligand 13 & + \\
\hline 10 & CCL17 & Chemokine (C-C motif) ligand 17 & - \\
\hline 11 & CCL18 & Chemokine (C-C motif) ligand 18 & - \\
\hline 12 & CCL19 & Chemokine (C-C motif) ligand 19 & - \\
\hline 13 & CCL20 & Chemokine (C-C motif) ligand 20 & + \\
\hline 14 & CCL21 & Chemokine (C-C motif) ligand 21 & + \\
\hline 15 & CCL22 & Chemokine (C-C motif) ligand 22 & - \\
\hline 16 & CCL24 & Chemokine (C-C motif) ligand 24 & - \\
\hline 17 & CXCL1 & Chemokine (C-X-C motif) ligand 1 & + \\
\hline 18 & CXCL2 & Chemokine (C-X-C motif) ligand 2 & + \\
\hline 19 & CXCL5 & Chemokine (C-X-C motif) ligand 5 & + \\
\hline 20 & CXCL9 & Chemokine (C-X-C motif) ligand 9 & - \\
\hline 21 & CXCL10 & Chemokine (C-X-C motif) ligand 10 & + \\
\hline 22 & CXCL11 & Chemokine (C-X-C motif) ligand 11 & + \\
\hline 23 & CXCL12 & Chemokine (C-X-C motif) ligand 12 & + \\
\hline 24 & CXCL13 & Chemokine (C-X-C motif) ligand 13 & - \\
\hline 25 & CXCL16 & Chemokine (C-X-C motif) ligand 16 & + \\
\hline 26 & CX3CL1 & Chemokine (C-X3-C motif) ligand 1 & + \\
\hline 27 & GPI/AMF & Glucose-6-phosphate isomerase & + \\
\hline 28 & IFNA2 & Interferon, alpha 2 & + \\
\hline 29 & MIF & Macrophage migration inhibitory factor & + \\
\hline 30 & PPBP & Proplatelet basic protein (chemokine (C-X-C motif) ligand 7) & + \\
\hline 31 & SPP1 & Secreted phosphoprotein 1 & + \\
\hline 32 & $\mathrm{XCL} 1$ & Chemokine ( $\mathrm{C}$ motif) ligand 1 & - \\
\hline \multicolumn{4}{|c|}{ Growth factors } \\
\hline 33 & BMP2 & Bone morphogenetic protein 2 & + \\
\hline 34 & BMP4 & Bone morphogenetic protein 4 & + \\
\hline 35 & BMP6 & Bone morphogenetic protein 6 & + \\
\hline 36 & BMP7 & Bone morphogenetic protein 7 & - \\
\hline 37 & CNTF & Ciliary neurotrophic factor & + \\
\hline 38 & CSF1 & Colony stimulating factor 1 (macrophage) & + \\
\hline 39 & CSF2 & Colony stimulating factor 2 (granulocyte-macrophage) & + \\
\hline 40 & CSF3 & Colony stimulating factor 3 (macrophage) & + \\
\hline 41 & LIF & Leukemia inhibitory factor (cholinergic differentiation factor) & + \\
\hline 42 & MSTN & Myostatin & + \\
\hline 43 & NODAL & Nodal homolog (mouse) & + \\
\hline 44 & OSM & Oncostatin M & + \\
\hline
\end{tabular}


TABle 1: Continued.

\begin{tabular}{|c|c|c|c|}
\hline Number & Symbol & Description & Detectable \\
\hline 45 & TGFB2 & Transforming growth factor, beta 2 & + \\
\hline 46 & THPO & Thrombopoietin & - \\
\hline 47 & VEGFA & Vascular endothelial growth factor A & + \\
\hline \multicolumn{4}{|c|}{ TNF superfamily } \\
\hline 48 & CD40LG & CD40 ligand & - \\
\hline 49 & FASLG & Fas ligand (TNF superfamily, member 6) & - \\
\hline 50 & LTA & Lymphotoxin alpha (TNF superfamily, member 1) & - \\
\hline 51 & LTB & Lymphotoxin beta (TNF superfamily, member 3) & + \\
\hline 52 & TNF & Tumor necrosis factor & + \\
\hline 53 & TNFRSF11B & Tumor necrosis factor receptor superfamily, member $11 \mathrm{~b}$ & + \\
\hline 54 & TNFSF10 & Tumor necrosis factor (ligand) superfamily, member 10 & + \\
\hline 55 & TNFSF11 & Tumor necrosis factor (ligand) superfamily, member 11 & - \\
\hline 56 & TNFSF13B & Tumor necrosis factor (ligand) superfamily, member 13b & + \\
\hline \multicolumn{4}{|c|}{ Interleukins } \\
\hline 57 & IL1A & Interleukin 1 , alpha & + \\
\hline 58 & IL1B & Interleukin 1 , beta & + \\
\hline 59 & IL1RN & Interleukin 1 receptor antagonist & + \\
\hline 60 & IL2 & Interleukin 2 & + \\
\hline 61 & IL3 & Interleukin 3 (colony-stimulating factor, multiple) & - \\
\hline 62 & IL4 & Interleukin 4 & - \\
\hline 63 & IL5 & Interleukin 5 (colony-stimulating factor, eosinophil) & - \\
\hline 64 & IL6 & Interleukin 6 (interferon, beta 2) & + \\
\hline 65 & IL7 & Interleukin 7 & + \\
\hline 66 & IL8 & Interleukin 8 & + \\
\hline 67 & IL9 & Interleukin 9 & - \\
\hline 68 & IL10 & Interleukin 10 & - \\
\hline 69 & IL11 & Interleukin 11 & + \\
\hline 70 & IL12A & Interleukin $12 \mathrm{~A}$ & + \\
\hline 71 & IL12B & Interleukin $12 \mathrm{~B}$ & - \\
\hline 72 & IL13 & Interleukin 13 & - \\
\hline 73 & IL15 & Interleukin 15 & + \\
\hline 74 & IL16 & Interleukin 16 & - \\
\hline 75 & IL17A & Interleukin $17 \mathrm{~A}$ & - \\
\hline 76 & IL17F & Interleukin $17 \mathrm{~F}$ & - \\
\hline 77 & IL18 & Interleukin 18 (interferon-gamma-inducing factor) & + \\
\hline 78 & IL21 & Interleukin 21 & - \\
\hline 79 & IL23A & Interleukin 23, alpha subunit p19 & + \\
\hline 80 & IL22 & Interleukin 22 & - \\
\hline 81 & IL24 & Interleukin 24 & - \\
\hline 82 & IL27 & Interleukin 27 & + \\
\hline \multicolumn{4}{|c|}{ Cytokines } \\
\hline 83 & ADIPOQ & Adiponectin, C1Q and collagen domain containing & - \\
\hline 84 & IFNG & Interferon, gamma & - \\
\hline
\end{tabular}

corresponding cell growth properties. The aim of this analysis was to identify cell growth-associated cytokines and chemokines by comparison of malignant and nonmalignant expression patterns, particularly with regard to the identification of putative biomarkers for RCC progression.

\section{Materials and Methods}

2.1. Cell Culture. RCC cell lines Caki-1, 786-O, A498 (Cell Lines Service, Eppelheim, Germany), and RCC4 (SigmaAldrich, München, Germany) and the nonmalignant renal cell 


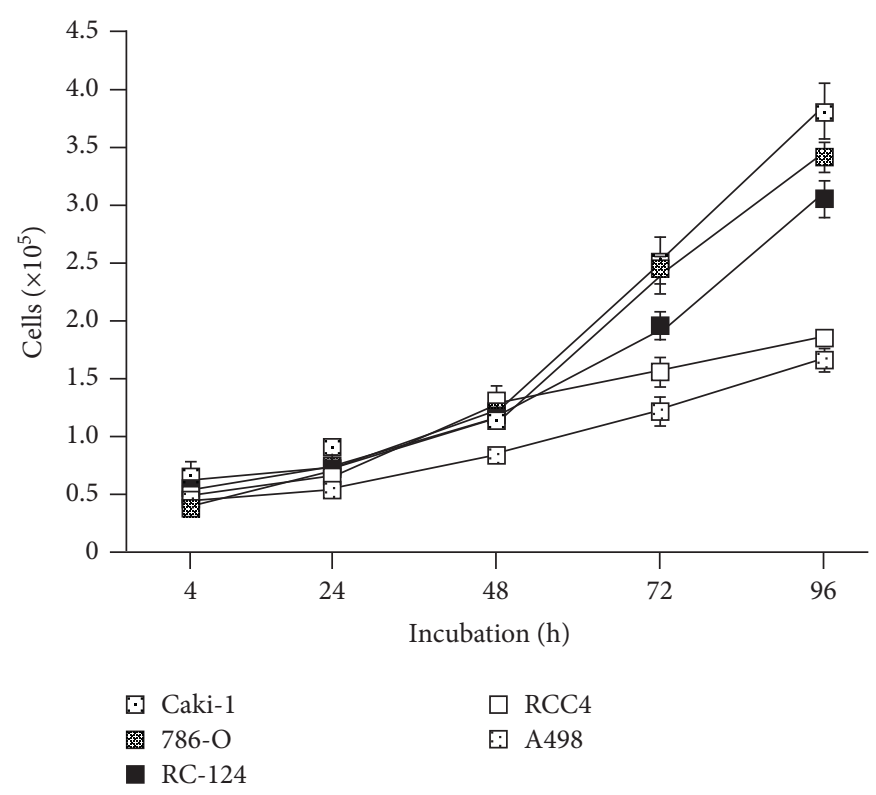

Figure 1: Cellular growth of the RCC cell lines Caki-1, 786-O, RCC4, and A498 as well as the nonmalignant renal cell line RC-124. Cellular growth was examined at indicated time points utilizing a CASY Cell Counter and Analyzer Model TT (Roche Applied Science). Results are expressed as the mean \pm SD of cell count.

line RC-124 (Cell Lines Service) were used. Caki-1 and A498 cells were cultivated in a minimum essential medium (MEM) supplemented with $79.6 \mathrm{mg} / \mathrm{l}$ nonessential amino acids, $2 \mathrm{mM}$ L-glutamine, $1 \mathrm{mM}$ sodium pyruvate, $1 \%$ penicillin/streptomycin $(\mathrm{P} / \mathrm{S})$, and $10 \%$ FBS (PAN Biotech, Aidenbach, Germany). 786-O cells were propagated in RPMI 1640 medium containing $2 \mathrm{mM}$ L-glutamine, $1 \% \mathrm{P} / \mathrm{S}$, and $10 \% \mathrm{FBS}$ (PAN Biotech); RCC4 cells were cultivated in Dulbecco's Modified Eagle Medium (DMEM) with $1 \mathrm{mM}$ sodium pyruvate, $1 \% \mathrm{P} / \mathrm{S}$, and $10 \%$ FBS (PAN Biotech); and RC-124 cells were cultivated in McCoy's 5a medium supplemented with $2 \mathrm{mM}$ L-glutamine, $1 \% \mathrm{P} / \mathrm{S}$, and $10 \% \mathrm{FBS}$ (PAN Biotech). All cells were propagated in a humidified atmosphere $\left(37^{\circ} \mathrm{C}, 5 \% \mathrm{CO}_{2}\right)$.

2.2. Proliferation Assay. Proliferation of cells was examined by cell counting (CASY Cell Analyzer, Roche Applied Science, Mannheim, Germany). Adherent cells, detached by trypsin/ethylenediaminetetraacetic acid (EDTA) treatment, were suspended in CASYton (Roche Applied Science) as 1:100 dilution. Measurement was performed using a capillary of $150 \mu \mathrm{m}$ in diameter and cell line-specific gate settings to discriminate between living cells, dead cells, and cellular debris (Caki-1: $8.57 \mu \mathrm{m} / 15.4 \mu \mathrm{m}, 786-\mathrm{O}: 6.9 \mu \mathrm{m} / 14.7 \mu \mathrm{m}$, RCC4: $8.5 \mu \mathrm{m} / 15.75 \mu \mathrm{m}, \mathrm{A}-498: 7.2 \mu \mathrm{m} / 15.75 \mu \mathrm{m}$, and RC124: $6.5 \mu \mathrm{m} / 12.75 \mu \mathrm{m})$.

2.3. Quantitative DNA Array. RCC cells and nonmalignant RC-124 cells were grown on a 6-well cell culture plate to $80 \%$ confluency and total RNA was prepared (peqGOLD TriFast reagent, Peqlab Biotechnology, Erlangen, Germany). Analysis of 84 human cytokines and chemokines (Table 1) was performed using the Human Cytokines \& Chemokines RT $^{2}$ Profiler PCR Array (Qiagen, Hilden, Germany) according to the supplier's instructions (http://www.sabiosciences.com).
Briefly, $0.5 \mu \mathrm{g}$ of total RNA was reversely transcribed and polymerase chain reaction (PCR) was done on a CFX96 Real-Time System (Bio-Rad, Munich, Germany) and analyzed with the CFX Manager software (Bio-Rad).

2.4. Enzyme-Linked Immunosorbent Assay (ELISA). IL6, IL8, IL11, IL15, and VEGF concentrations in cell culture supernatant of RCC cells and nonmalignant RC-124 cells were determined by use of target-specific DuoSet ELISA kits with a Substrate Reagent Pack containing stabilized hydrogen peroxide and tetramethylbenzidine (all R\&D, Minneapolis, MN, USA) according to the supplier's instructions. Cells were sedimented $(1.3 \times \mathrm{g}, 5 \mathrm{~min})$, the supernatant was incubated with capture antibodies (overnight, $4^{\circ} \mathrm{C}$ ), and soluble antigens were determined applying the BMG FLUOstar OPTIMA Microplate Reader with OPTIMA software 2.10 (BMG Labtech, Offenbach, Germany). Each sample was analyzed in duplicates.

2.5. Statistics. For data evaluation, the graphics and statistics software Graph Pad Prism V5.01 (GraphPad Software, La Jolla, CA, USA) was used. Principal component analysis (PCA) was performed using Multibase 2015 software (NumericalDynamics.Com, Tokyo, Japan).

\section{Results}

In contrast to the elevated proliferation rates of Caki- 1 and 786-O cells compared to those of nonmalignant RC-124 cells, both, RCC4 and A498 cells, showed a markedly reduced cell growth (Figure 1). These differences in proliferation prompted us to investigate whether cytokines and chemokines may control the individual growth properties of the RCC cell lines.

Performing the $\mathrm{RT}^{2}$ Profiler PCR Array Human Cytokines \& Chemokines, the expression of 84 target mRNAs 
IL6

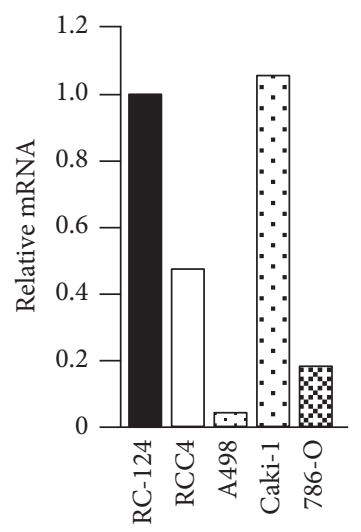

(a)

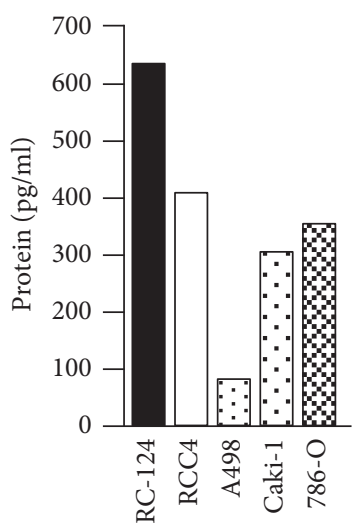

L11
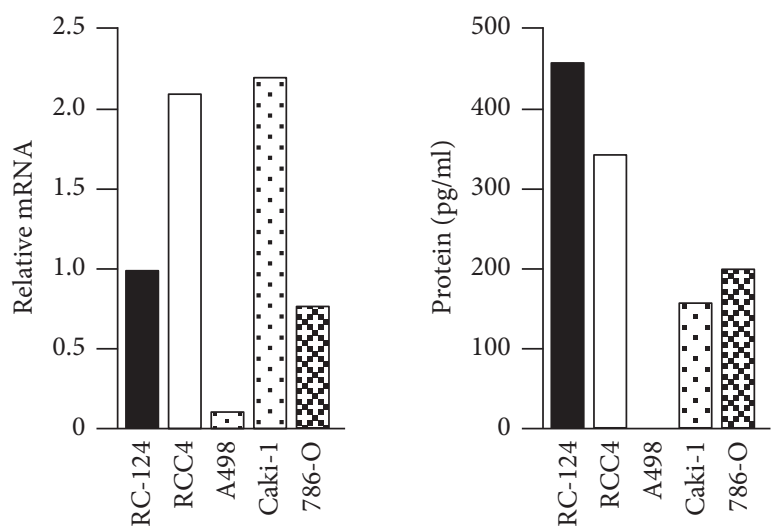

(c)

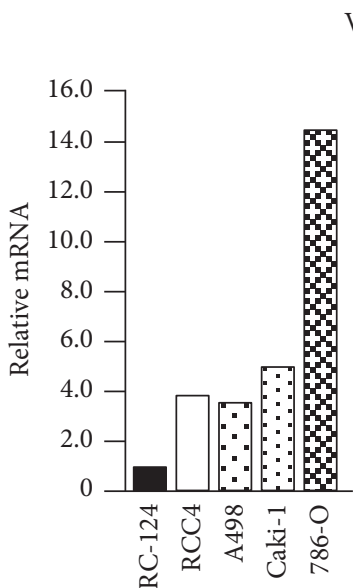

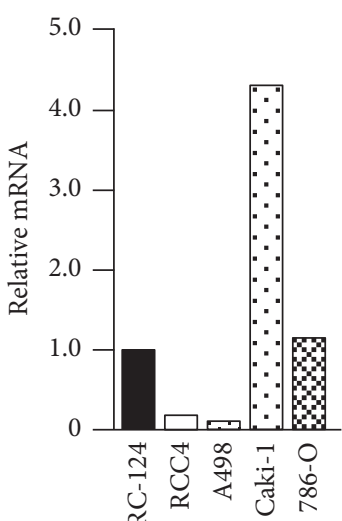

IL8

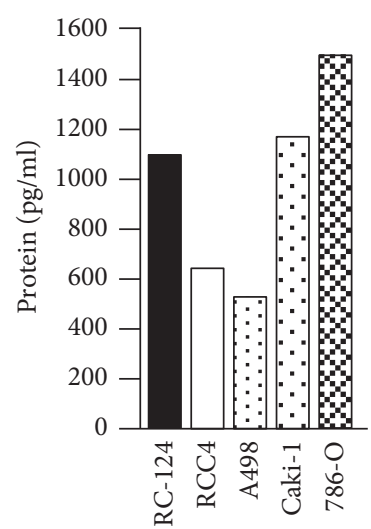

(b)

IL15
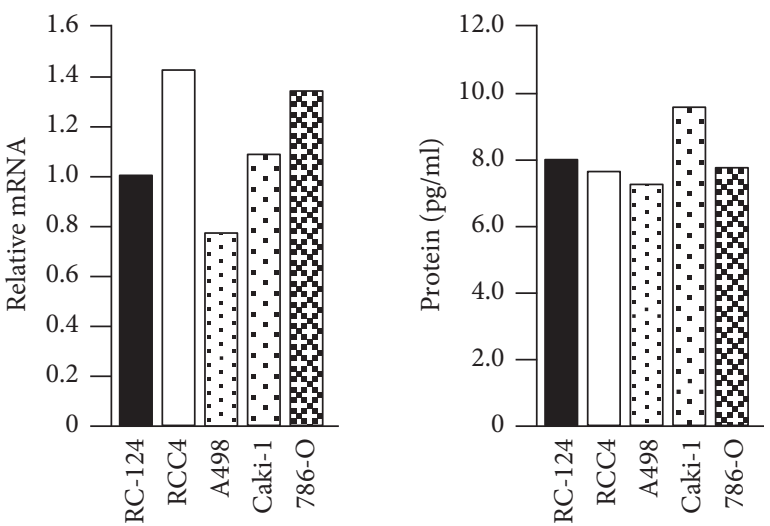

(d)

VEGF

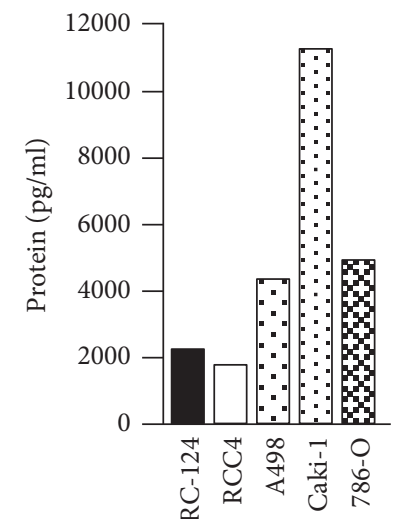

(e)

Figure 2: Comparison of mRNA levels and protein levels of selected cytokines. Steady-state levels of intracellular mRNA levels and extracellular protein levels of IL6 (a), IL8 (b), IL11 (c), IL15 (d), and VEGF (e) were assessed by quantitative DNA array (Human Cytokines \& Chemokines $\mathrm{RT}^{2}$ Profiler PCR Array; Qiagen) and enzyme-linked immunosorbent assay (ELISA) analysis, respectively. mRNA amounts are expressed as relative mRNA levels normalized to nonmalignant RC-124 cells $(\mathrm{RC}-124 \mathrm{mRNA}=1.0)$. Protein levels are expressed as pg protein $/ \mathrm{ml}$ cell culture supernatant.

(Table 1) was measured. From these 84 factors, which have been examined at the intracellular mRNA level, the 5 cytokines IL6, IL8, IL11, IL15, and VEGF have been exemplarily chosen for verification at the extracellular protein level via
ELISA. mRNA concentrations of IL6, IL8, IL11, IL15, and VEGF appeared to correspond to the levels of secreted proteins of the RCC cells and the nonmalignant renal cell line (Figure 2). The transcript pattern of IL6, IL8, and IL15 was 


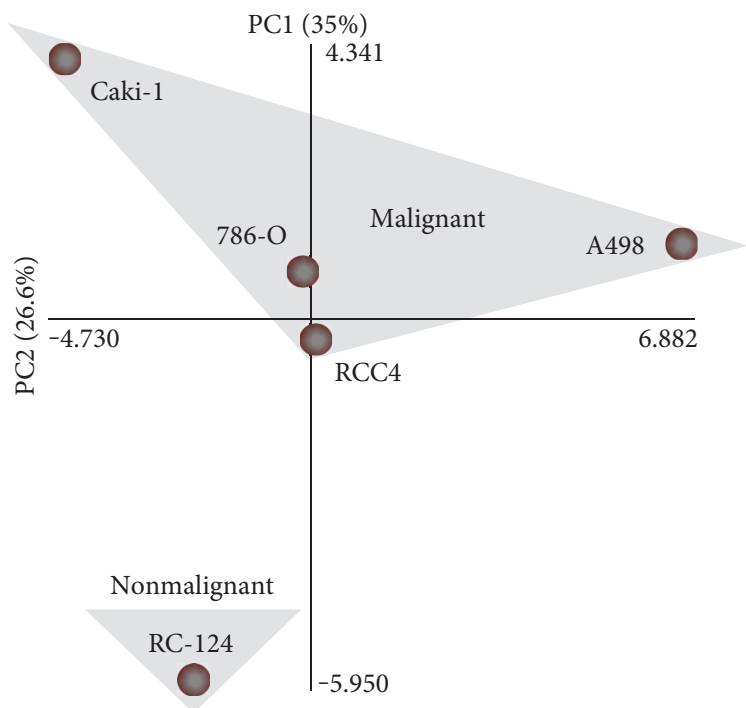

FIGURE 3: Principal component analysis (PCA) of the cytokine and chemokine expression. The RCC cell lines Caki-1, 786-O, RCC4, and A498 as well as the nonmalignant renal cell line RC-124 were plotted in a two-dimensional coordinate plane defined by the principal components 1 (PC1) and 2 (PC2) analyzing the expression data of all cytokines and chemokines measured by the DNA array (Human Cytokines \& Chemokines RT $^{2}$ Profiler PCR Array; Qiagen). Clustering of the malignant cells is indicated by a grey triangle.

very comparable to the levels of secreted proteins in all cell lines. However, there were differences in Caki-1 cells with respect to the low level of secreted IL11 protein and the high level of secreted VEGF protein compared to that of intracellular mRNA. Exactly opposite results were found with respect to 786-O cells. Nevertheless, comparison of cytokines' mRNA and protein levels revealed a clear correlation indicating mRNA arrays' applicability for expression analyses of cytokines and chemokines.

Furthermore, a global normalized PCA was performed and used as quality check. PCA was carried out on the concentrations of all of the detected cytokines and chemokines in all of the five cell lines (Figure 3 ) dividing cancer and noncancer cells (PC1) and defining individual characteristics of all cell lines (PC2). As expected, the five cell lines used in this study were divided correctly into two major groups corresponding to malignant versus nonmalignant physiology.

Performing the Human Cytokines \& Chemokines RT $^{2}$ Profiler PCR Array, the expression of various cytokines and chemokines for each of the cell lines was analyzed compared to the nonmalignant cell line RC-124. The following 32 cytokines and chemokines were not expressed: ADIPQ, BMP7, CCL1, CCL17, CCL18, CCL19, CCL22, CCL24, CCL3, CCL8, CD40LG, CXCL13, CXCL9, FASLG, IFNG, IL10, IL12B, IL13, IL16, IL17A, IL17F, IL21, IL22, IL24, IL3, IL4, IL5, IL9, LTA, THPO, TNFSF11, and XCL1.

52 factors were modulated compared to RC-124 cells (Figure 4). The group of chemokines (Figure 4(a)) was dominated by the overexpression of CCL20, CXCL10, and foremost CCL5 with more than a 100-fold overexpression. Most striking was the strong upregulation of these three chemokines in A498 cells, even compared to the other RCC cell lines. Within the family of growth factors, a more balanced distribution pattern of the factors was demonstrated (Figure 4(b)). However, only CNTF, CSF1, CSF3, LIF,
MSTN, and VEGFA were detectable in all of the four malignant cell lines.

Five members of the TNF superfamily were detected (Figure 4(c)); however, only TNFSF13B was strongly overexpressed in all of the four malignant cell lines, dominated by an upto 25-fold overexpression in Caki-1 and RCC4 cells. Considering the family of interleukins (Figure 4(d)), a total of 13 different factors were detected with an upregulated expression of the interleukins IL1A, IL1B, and IL1RN in each of the 4 malignant cell lines.

\section{Discussion}

The process of tumorigenesis is driven by several mechanisms including an increased proliferation, angiogenesis, and immunomodulation [13]. Cytokines and chemokines, in general, are primarily regulators of immune defense. CCL5, in particular, supports leukocyte diapedesis by integrin induction $[14,15]$. Interestingly, CCL5 expression in all four RCC cell lines was highly upregulated. Since tumorigenesis is associated with inflammatory response, elevated CCL5 secretion by RCC cells may be part of cancer-related inflammation. Corresponding to this observation is the finding that the expression levels of proinflammatory interleukins IL1A and IL1B are increased in all of the four RCC cell lines. Interestingly, overexpression of the anti-inflammatory IL1RN [16] could only be observed in the lowly proliferative RCC4 and A498 cells. This could mean that IL1RN may interfere with antiproliferative pathways or that anti-inflammatory processes generally attenuate cell growth.

Within the TNF superfamily, TNFSF13B is significantly overexpressed in all four RCC cell lines. Looking at the large differences in cellular growth of highly proliferative Caki-1 and 786-O and lowly proliferative RCC4 and A498 cell lines, however, classification of TNFSF13B as a proliferative factor 


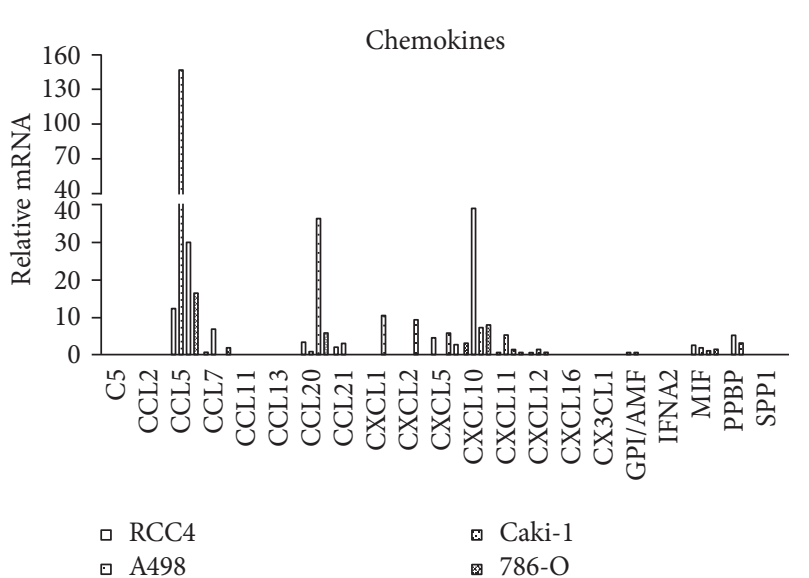

(a)

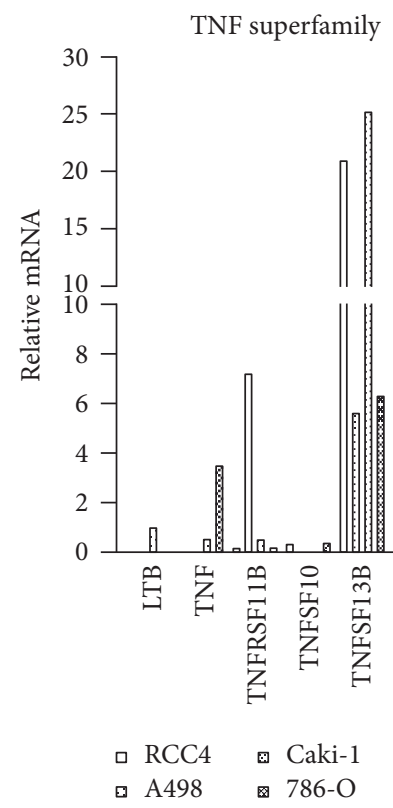

(c)

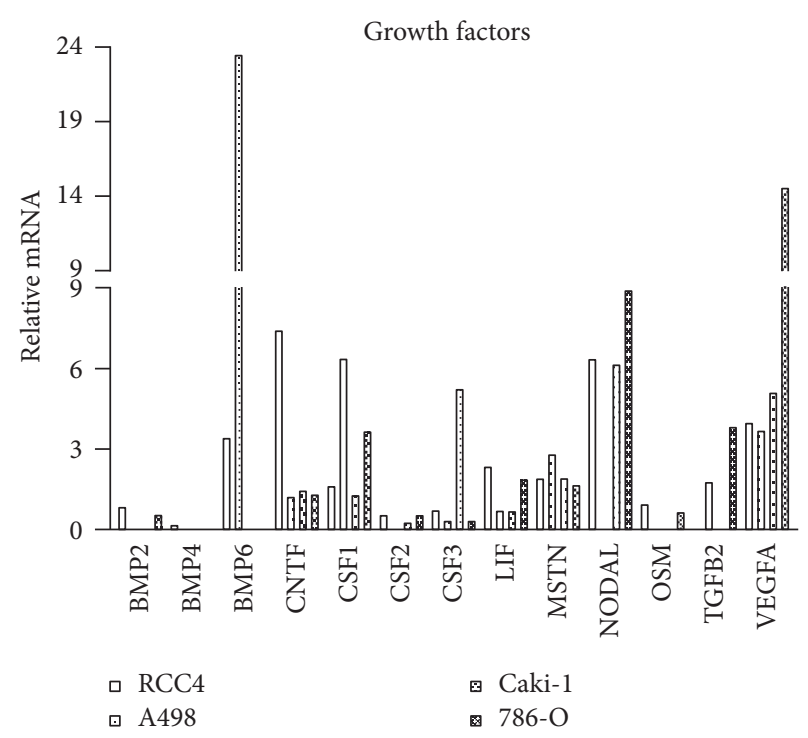

(b)

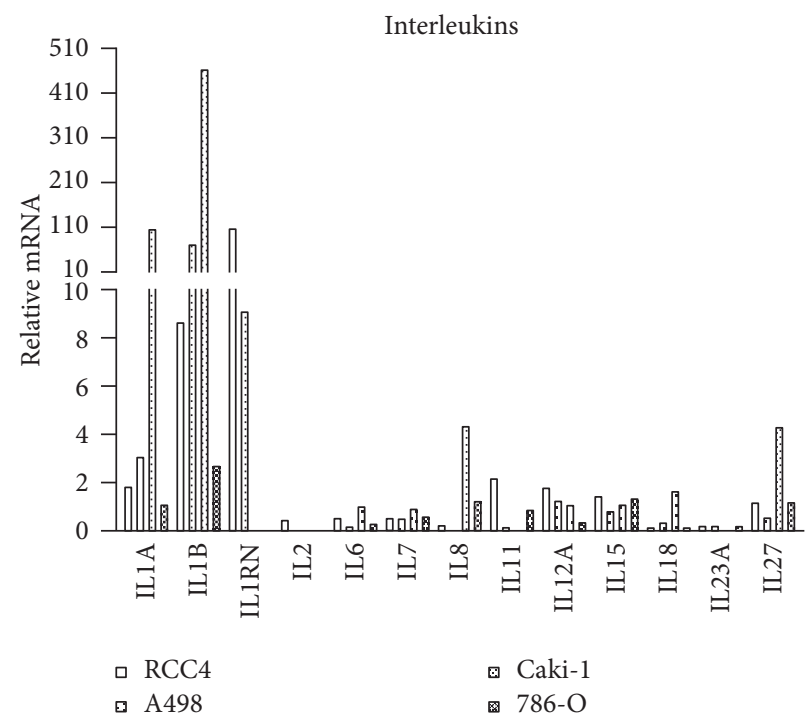

(d)

FIGURE 4: Expression profile of cytokines and chemokines in RCC cell lines. Expression data of cytokines and chemokines in the RCC cell lines Caki-1, 786-O, RCC4, and A498 as well as in the nonmalignant renal cell line RC-124 were measured performing a DNA array (Human Cytokines \& Chemokines RT ${ }^{2}$ Profiler PCR Array; Qiagen) and expressed as relative mRNA normalized to nonmalignant RC124 cells $(\mathrm{RC}-124=1.0)$. Cytokines and chemokines were classified into the four groups of chemokines (a), growth factors $(\mathrm{b})$, TNF superfamily members (c), and interleukins (d).

in RCC, as has been done in previous studies [17], could not be clearly confirmed.

As expected, expression levels of proliferative cytokines, for example, CSF-1, CSF-3, NODAL, and VEGFA, correlated with the cell growth rates of the highly proliferative (Caki-1, 786-O) and the lowly proliferative (RCC4, A498) RCC cells. This observation has been confirmed by several studies. CSF- 1 and VEGFA expression is associated with RCC progression and bad prognosis for patient survival $[11,18,19]$. Furthermore, elevated levels of NODAL, a member of the TGF $\beta$ superfamily, confer enhanced tumorigenicity
[20-23]. Expectedly, strongly overexpressed proliferative factors, for example, VEGFA and NODAL, may represent novel prognostic markers for RCC.

Vice versa, the expression of BMP6 was significantly increased in the lowly proliferative cell lines RCC4 and A498, whereas an expression in highly proliferative Caki-1 and 786-O cells was not detectable. Since BMP6 is considered to be a tumor suppressor in breast cancer [24], upregulation of BMP6 in RCC cells may contribute to attenuated cell growth of RCC4 and A498 cells compared to that of the nonmalignant cell line RC-124. 
Taken together, there are a number of cytokines and chemokines which are differentially expressed not only in malignant and nonmalignant renal cells but also in the four RCC cell lines. These results may reflect heterogeneity among RCC patients as well as among RCC subtypes. In addition, this may suggest a functional substitution of members of the cytokine/chemokine system due to an activation of alternative factors involved in cytokine/chemokine signalling. Notably, two proliferative factors (VEGFA, NODAL) and a tumor suppressive factor (BMP6) have been found which may significantly contribute to the malignant phenotype of RCC and thus may be suitable as novel marker proteins in RCC diagnosis and therapy.

\section{Conflicts of Interest}

The authors have no conflicts of interest to disclose.

\section{Authors' Contributions}

Nadine Gelbrich and Hannes Ahrend contributed equally to this study.

\section{Acknowledgments}

The authors thank Katja Wittig for her excellent technical assistance.

\section{References}

[1] J. S. Parihar and H. S. G. R. Tunuguntla, "Role of chemokines in renal cell carcinoma," Reviews in Urology, vol. 16, pp. 118121, 2014.

[2] G. Low, G. Huang, W. Fu, Z. Moloo, and S. Girgis, "Review of renal cell carcinoma and its common subtypes in radiology," World Journal of Radiology, vol. 8, no. 5, pp. 484-500, 2016.

[3] K. L. Reckamp, R. M. Strieter, and R. A. Figlin, "Chemokines as therapeutic targets in renal cell carcinoma," Expert Review of Anticancer Therapy, vol. 8, no. 6, pp. 887-893, 2008.

[4] M. Lahn, P. Fisch, G. Köhler et al., "Pro-inflammatory and T cell inhibitory cytokines are secreted at high levels in tumor cell cultures of human renal cell carcinoma," European Urology, vol. 35, no. 1, pp. 70-80, 1999.

[5] Q. Xu, M. Krause, A. Samoylenko, and S. Vainio, "Wnt signaling in renal cell carcinoma," Cancers (Basel), vol. 8, no. 6 , p. $57,2016$.

[6] V. Levina, B. M. Nolen, A. M. Marrangoni et al., "Role of eotaxin-1 signaling in ovarian cancer," Clinical Cancer Research, vol. 15, no. 8, pp. 2647-2656, 2009.

[7] M. Mo, M. Zhou, L. Wang et al., "CCL21/CCR7 enhances the proliferation, migration, and invasion of human bladder cancer T24 cells," PLoS One, vol. 10, no. 3, article e0119506, 2015.

[8] J. Mei and L. Liu, "Role of interleukin 17 in lung carcinogenesis and lung cancer progression," Chinese Journal of Lung Cancer, vol. 19, no. 1, pp. 46-51, 2016.

[9] D. Wu, P. Wu, Q. Huang, Y. Liu, J. Ye, and J. Huang, "Interleukin-17: a promoter in colorectal cancer progression," Clinical and Developmental Immunology, vol. 2013, Article ID 436307, 7 pages, 2013.

[10] T. Welte and X. H. F. Zhang, "Interleukin-17 could promote breast cancer progression at several stages of the disease,"
Mediators of Inflammation, vol. 2015, Article ID 804347, 6 pages, 2015.

[11] F. C. Zeng, M. Q. Zeng, L. Huang et al., "Downregulation of VEGFA inhibits proliferation, promotes apoptosis, and suppresses migration and invasion of renal clear cell carcinoma," Journal of Onco Targets and Therapy, vol. 9, pp. 2131-2141, 2016.

[12] R. Lu, Z. Ji, X. Li et al., “Tumor suppressive microRNA-200a inhibits renal cell carcinoma development by directly targeting TGFB2," Tumor Biology, vol. 36, no. 9, pp. 6691-6700, 2015.

[13] C. Dosquet, M. C. Coudert, E. Lepage, J. Cabane, and F. Richard, "Are angiogenic factors, cytokines, and soluble adhesion molecules prognostic factors in patients with renal cell carcinoma?," Clinical Cancer Research, vol. 3, pp. 2451-2458, 1997.

[14] A. M. Krensky and Y. T. Ahn, "Mechanisms of disease: regulation of RANTES (CCL5) in renal disease," Nature Clinical Practice Nephrology, vol. 3, no. 3, pp. 164-170, 2007.

[15] S. Lin, S. Wan, L. Sun et al., "Chemokine C-C motif receptor 5 and $\mathrm{C}-\mathrm{C}$ motif ligand 5 promote cancer cell migration under hypoxia," Cancer Science, vol. 103, no. 5, pp. 904-912, 2012.

[16] J. Banchereau, V. Pascual, and A. O'Garra, "From IL-2 to IL37: the expanding spectrum of anti-inflammatory cytokines," Nature Immunology, vol. 13, no. 10, pp. 925-931, 2012.

[17] V. Pelekanou, G. Notas, K. Theodoropoulou et al., "Detection of the TNFSF members BAFF, APRIL, TWEAK and their receptors in normal kidney and renal cell carcinomas," Analytical Cellular Pathology (Amsterdam), vol. 34, no. 1-2, pp. 49-60, 2011.

[18] W. Yu, J. Chen, Y. Xiong, F. J. Pixley, Y. G. Yeung, and E. R. Stanley, "Macrophage proliferation is regulated through CSF-1 receptor tyrosines 544, 559, and 807," The Journal of Biological Chemistry, vol. 287, no. 17, pp. 13694-13704, 2012.

[19] L. Yang, Q. Wu, L. Xu et al., "Increased expression of colony stimulating factor-1 is a predictor of poor prognosis in patients with clear-cell renal cell carcinoma," BMC Cancer, vol. 15, p. 67, 2015.

[20] N. J. Kenney, H. B. Adkins, and M. Sanicola, "Nodal and Cripto-1: embryonic pattern formation genes involved in mammary gland development and tumorigenesis," Journal of Mammary Gland Biology and Neoplasia, vol. 9, no. 2, pp. 133-144, 2004.

[21] L. Vallier, M. Alexander, and R. A. Pedersen, "Activin/Nodal and FGF pathways cooperate to maintain pluripotency of human embryonic stem cells," Journal of Cell Science, vol. 118, Part 19, pp. 4495-4509, 2005.

[22] M. G. Lawrence, N. V. Margaryan, D. Loessner et al., "Reactivation of embryonic nodal signaling is associated with tumor progression and promotes the growth of prostate cancer cells," The Prostate, vol. 71, no. 11, pp. 1198-1209, 2011.

[23] I. Papageorgiou, P. K. Nicholls, F. Wang et al., "Expression of nodal signaling components in cycling human endometrium and in endometrial cancer," Reproductive Biology and Endocrinology, vol. 7, p. 122, 2009.

[24] G. Liu, Y. J. Liu, W. J. Lian, Z. W. Zhao, T. Yi, and H. Y. Zhou, "Reduced BMP6 expression by DNA methylation contributes to EMT and drug resistance in breast cancer cells," Oncology Reports, vol. 32, no. 2, pp. 581-588, 2014. 


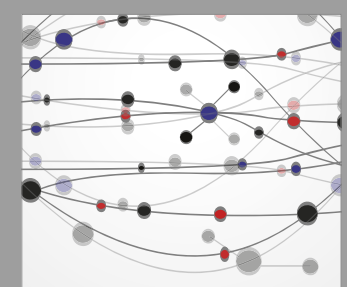

The Scientific World Journal
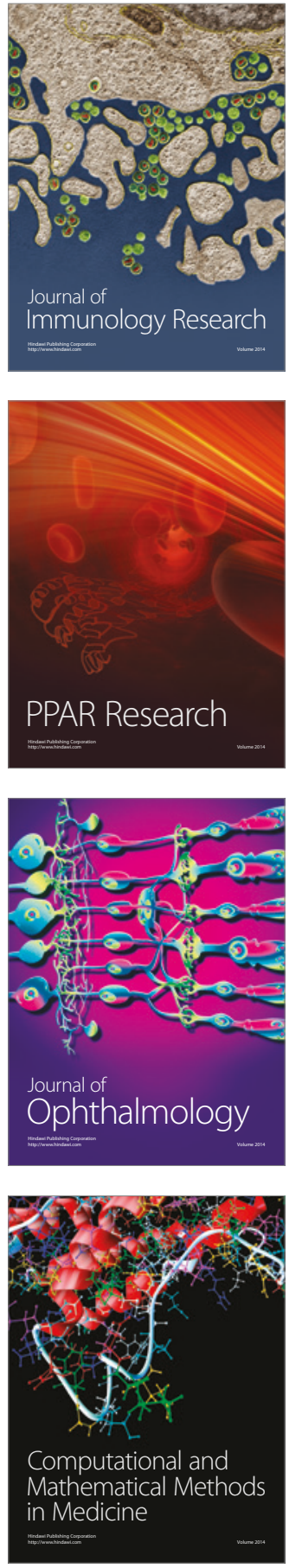

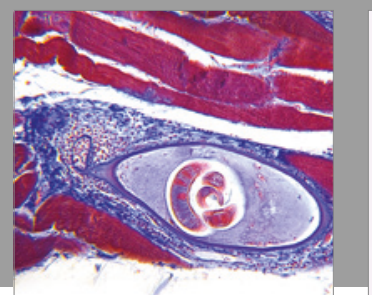

Gastroenterology Research and Practice
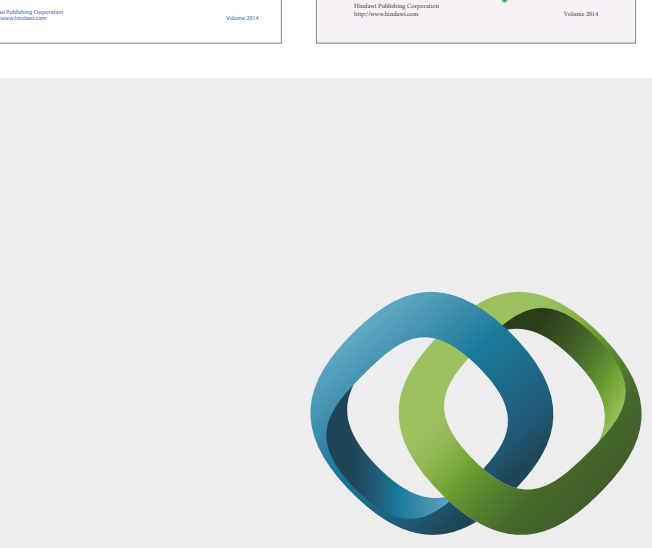

\section{Hindawi}

Submit your manuscripts at

https://www.hindawi.com
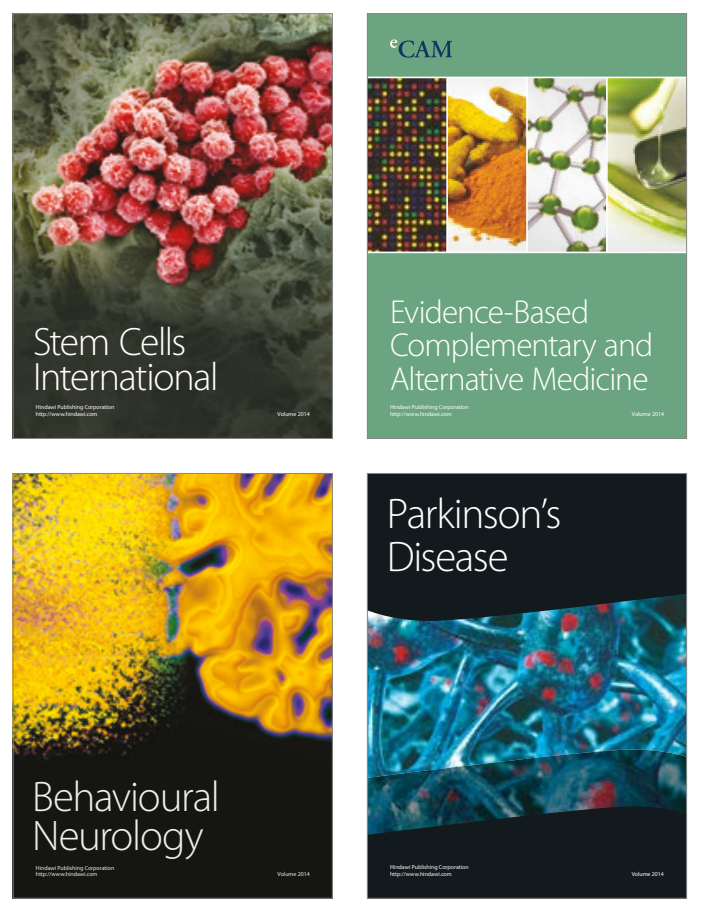
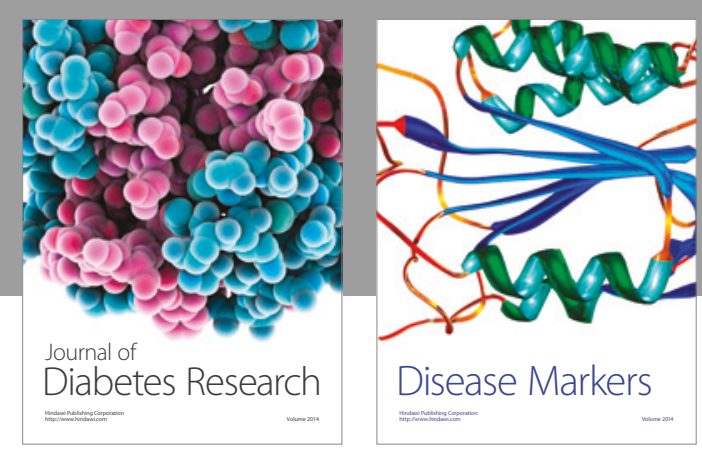

Disease Markers
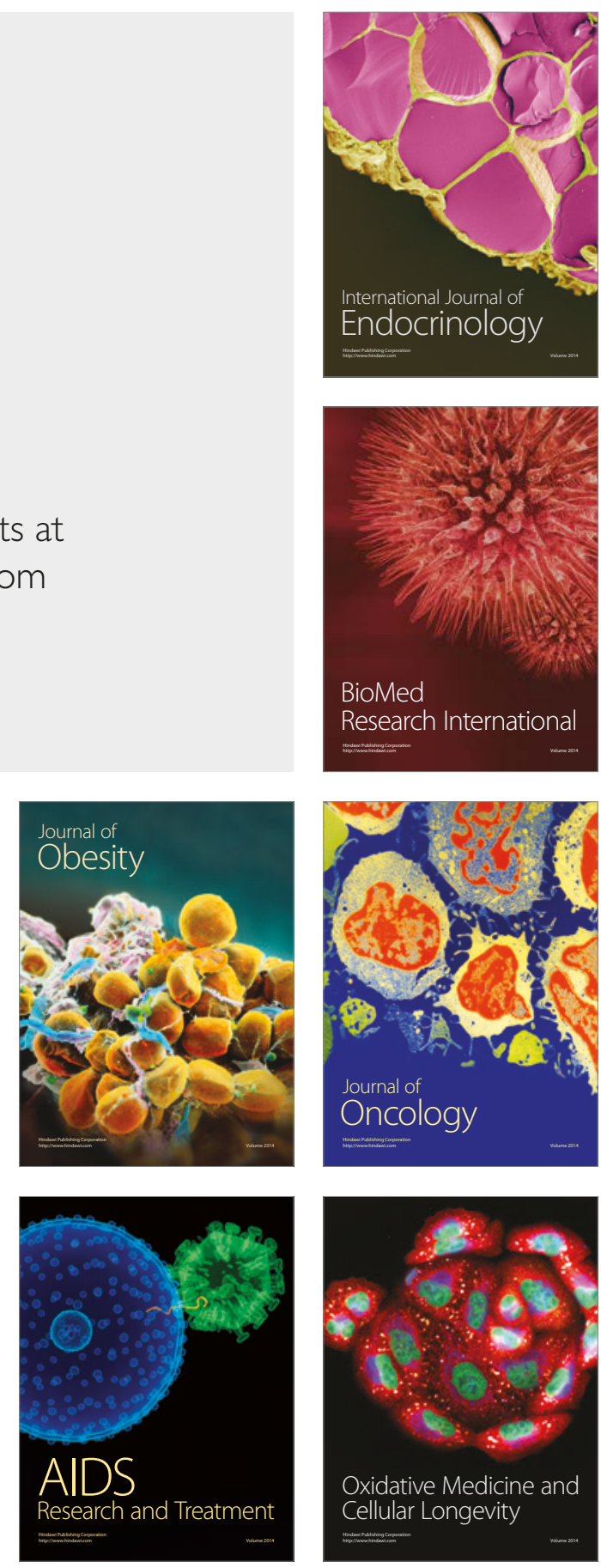